\section{Nouvelle lecture du bâton percé de la grotte du Trilobite (Arcy-sur-Cure, Yonne)}

La grotte du Trilobite fait partie d'un ensemble de cavernes situées sur la rive gauche de la Cure en amont d'Arcy-sur-Cure et qui ont toutes livré des vestiges archéologiques, de la préhistoire aux périodes historiques. Étudiées depuis 1850, elles font l'objet, à la fin du XIX ${ }^{\mathrm{e}}$ siècle, des recherches d'archéologues locaux (A. Ficatier) puis des remarquables travaux de l'abbé Parat. En 1946, après la découverte des gravures de la grotte du Cheval par G. Méraville, A. Leroi-Gourhan et son équipe entreprennent de nouvelles recherches dans les grottes du massif. Jusqu'en 1963, les grottes du Loup, de l'Hyène, du Renne, du Lion et du Bison sont étudiées. La découverte de peintures préhistoriques dans la Grande Grotte par P. Guilloré en 1990 relance les recherches archéologiques sur le site d'Arcy-sur-Cure. Enfin, depuis 1995, la grotte du Bison fait l'objet de nouvelles recherches sous la direction de F. David.

Le bâton percé qui fait l'objet de cet article (fig. 1) a été mis au jour en 1886 par l'archéologue auxerrois Adrien Ficatier, inventeur de la grotte, qui le décrit ainsi : «Un bâton de commandement fracturé vers le trou; il ne présente en fait d'ornements que deux ou trois rainures encadrant la base du trou» (Ficatier, 1886). Le matériel archéologique a ensuite été vendu pour être incorporé dans la collection de l'abbé Parat mise au jour lors de la reprise des fouilles par ce dernier de 1895 à 1898 (Parat, 1902). L'objet en question porte le numéro d'inventaire TF 18 (Parat, 1903). Sa position stratigraphique est clairement décrite par A. Ficatier et confirmée ensuite par l'abbé Parat: il s'agit de la couche 5 magdalénienne, notamment connue pour avoir livré deux pendeloques, un trilobite fossile ayant donné son nom à la grotte et un bupreste en jais, tous deux associés, selon A. Ficatier, à du matériel lithique magdalénien.

La collection complète des objets provenant des fouilles de la grotte du Trilobite (Parat et Ficatier) a été déposée au musée du petit séminaire de Joigny, jusqu'à son transfert et dépôt actuel au musée de l'Avallonnais le 21 juillet 1980 grâce à l'action de P. Poulain, ancien conservateur de ce dernier musée. En 1992, ce bâton percé est inventorié dans les fiches typologiques de l'industrie osseuse préhistorique (Peltier, 1992, fig. 4) puis, en 1995, D. Baffier et M. Julien le décrivent à nouveau dans un article consacré aux occupations magdaléniennes d'Arcy-sur-Cure et Saint-Moré : «Il s'agit donc d'un décor très simple soulignant seulement la morphologie générale de la pièce » (Baffier et Julien, in Schmider et al., 1995).

En 2005, dans le cadre de la préparation d'une exposition consacrée au site d'Arcy-sur-Cure au musée de l'Avallonnais, P. Bodu fait transférer une partie du matériel des fouilles Parat dans les locaux de l'UMR 7041 ARSCAN. Le matériel lithique et osseux provenant, entre autres, de la grotte du Trilobite est photographié par S. Oboukhoff, puis il est confié à l'une d'entre nous (D. M.) pour être dessiné. L'examen attentif de l'objet TF 18 révèle alors une série de petits traits gravés à proximité de la perforation. Un léger dépoussiérage de la surface permet ensuite de reconnaître, puis d'identifier avec certitude, des traits organisés qui figurent un cervidé.

\section{Description technique (J.-M. P.)}

Le bâton percé est façonné sur un bois de renne droit, de petit module, provenant d'une femelle ou d'un jeune mâle. La partie utilisée comprend la perche A + B et la
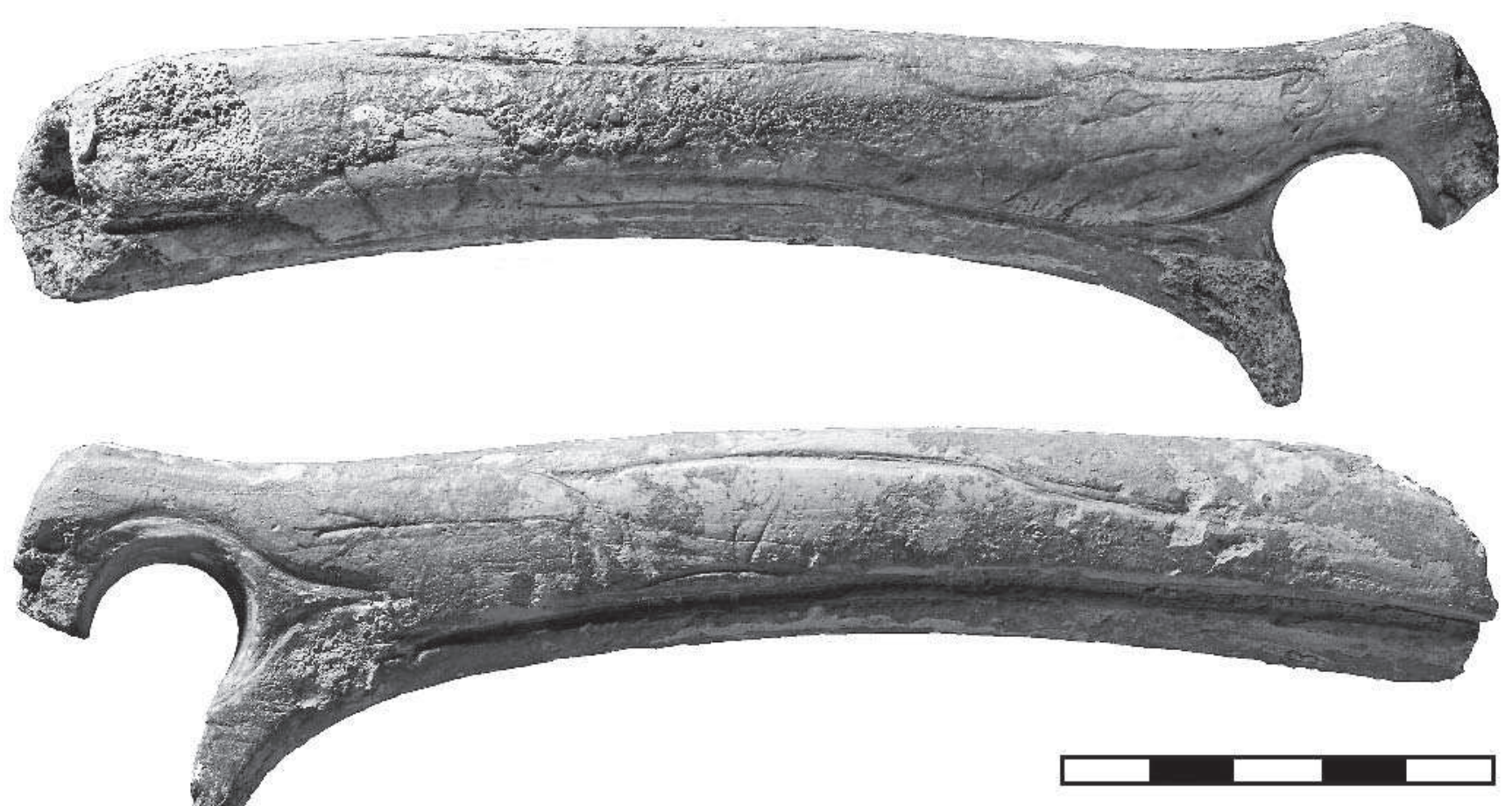

Fig. 1 - Bâton percé (photo S. Oboukhoff; l'orientation de l'objet privilégie la lecture du décor et non les conventions de la typologie osseuse). 
base de l'andouiller de glace. Pour obtenir le support, le bois a été sectionné au-dessus de la meule et au niveau de la perche $\mathrm{B}$, mais les traces de ces opérations ne sont plus visibles : l'extrémité distale (celle portant la perforation) est très émoussée, et l'extrémité proximale a subi des dommages récents (fracture postdépositionnelle) - ce qui implique d'ailleurs que l'objet était à l'origine plus long de ce côté. La composition du décor intègre manifestement la présence de la perforation (plusieurs traits gravés en soulignent le contour, la tête de cervidé est placée dans la zone laissée libre...); celle-ci a donc vraisemblablement été réalisée en premier. Un raclage a également été réalisé sur la face postérieure, sur une longueur d'environ 54 millimètres, visiblement dans le but d'aplanir cette face.

Il est difficile de déterminer si les fractures au niveau de la perforation résultent de l'utilisation de l'objet. La forme du pan de fracture situé côté distal signale un dégât post-dépositionnel. En revanche, le pan de fracture situé côté proximal présente une morphologie «en languette» qui pourrait indiquer une fracture sur bois frais : il pourrait donc s'agir ici d'un dégât survenu pendant l'utilisation.

Le fût de l'objet présente trois profondes rainures longitudinales qui peuvent difficilement s'intégrer au décor gravé. La première, longue de 123 millimètres, se trouve à la limite entre les faces antérieure et latérale du bois; les deux autres, parallèles et longues de 15 et 65 millimètres, sont à la limite entre les faces antérieure et médiale. Les trois rainures sont interrompues par la fracture distale. Les deux rainures les plus longues délimitent, sur la face antérieure du bois, un «bandeau» large de 11 à 12 millimètres. Cet ensemble de traces évoque clairement un début d'extraction de baguette par double rainurage longitudinal, conduit selon un schéma opératoire très fréquent au Magdalénien. Il semble par ailleurs que l'une des rainures (celle longue de 65 millimètres) recoupe une partie du décor. Il est donc vraisemblable que ce début d'extraction de baguette - qui n'a pas été mené jusqu'à son terme - ait été effectué après la mise en place du décor. Il est alors tentant de reconstituer le schéma suivant : après avoir été fabriqué, décoré, utilisé (et fracturé ?), le bâton percé a subi un début de recyclage comme matrice d'extraction de baguettes. Le recyclage d'un bâton percé en matrice de baguettes est un comportement déjà attesté au Magdalénien (par exemple dans le Magdalénien supérieur d'Isturitz - Pétillon, 2006, p. 187 ou dans le Magdalénien du Grand-Pastou - Dachary et Chauvière, 2006, p. 179), mais jamais auparavant à notre connaissance sur un exemplaire décoré.
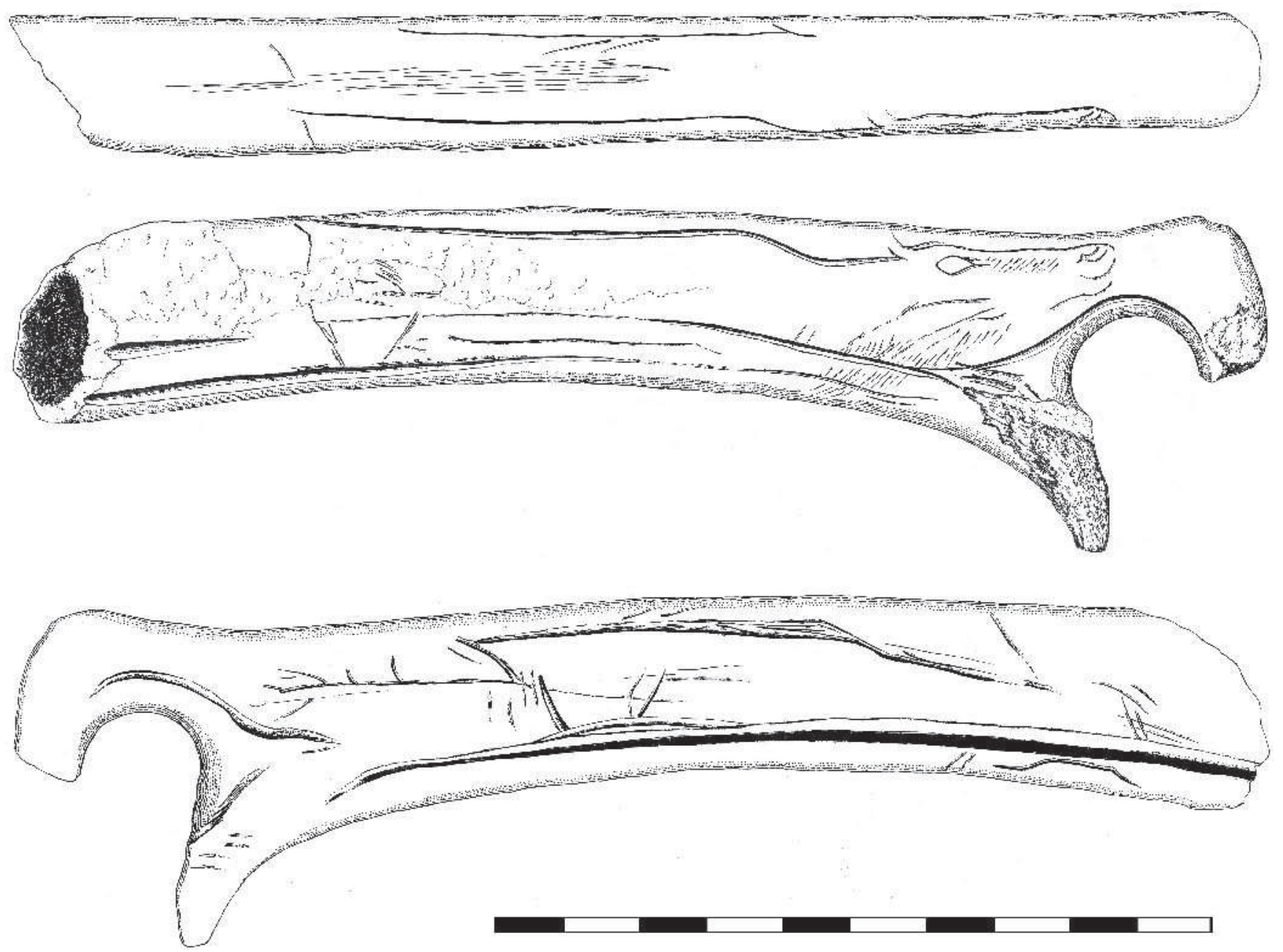

Fig. 2 - Relevé du bâton percé (dessin D. Molez). 


\section{Le décor (D. M.)}

Les deux faces sont gravées (fig. 2).

Première face : il s'agit de la figuration d'un cervidé en profil droit. La ligne de dos est constituée d'un trait largement incisé, se prolongeant par une encolure tendue, la tête projetée en arrière. On note la présence de deux petits traits (départ de ramure ?), le concrétionnement ne permettant pas de lire au-delà. L'œil, bien marqué, se prolonge pour former le larmier, une série de petites incisions assurant la jonction avec le mufle dont les naseaux sont finement représentés. Le trait inférieur de la bouche, bien que non jointif à la partie supérieure, ne semble pas indiquer une bouche ouverte. Un groupe d'incisions obliques figure les fanons de l'animal. Une seule cuisse est figurée et l'arrondi de la croupe se prolonge pour former l'amorce de la queue. La ligne de ventre, finement incisée, part de l'avant de la cuisse et se termine par ce qui pourrait être l'amorce de la patte avant. Au-dessus une ligne part de la cuisse, s'interrompt pour le trait interne de celle-ci, continue vers l'avant, s'incurve vers le bas pour rejoindre une rainure profonde qui suit le bord de la perforation. Son interruption au niveau de la cuisse montre qu'elle fait bien partie de l'ensemble gravé. Il semblerait que l'on soit en présence de la représentation d'un cervidé nageant, la tête redressée hors de l'eau, les fanons inclinés vers l'arrière, le trait passant sur la cuisse figurant la ligne d'eau.

Seconde face : elle est très concrétionnée et endommagée par la présence de rainures d'extraction de baguette postérieures à la gravure. On distingue une rainure profonde qui rappelle dans son dessin la ligne de dos de l'autre face; l'extrémité proximale de la pièce, très calcitée, ne permet pas l'identification. À l'extrémité distale, une incision incurvée semble figurer une croupe terminée par l'amorce de la queue. Plusieurs traits verticaux sont présents à l'emplacement présumé de la patte arrière. Un long trait horizontal, comportant des incisions perpendiculaires, fait penser à une flèche empennée fichée dans la croupe de l'animal. Malgré l'altération plus importante du dessin et le fait qu'il soit très calcité, il est tentant de voir une similitude entre les deux faces de l'objet.

Cet objet inédit est désormais une pièce majeure de l'art mobilier paléolithique d'Arcy-sur-Cure. Les peintures et les gravures de la Grande Grotte ont replacé le site d'Arcy-sur-Cure au cœur des débats sur les manifestations artistiques, réputées peu nombreuses, des populations du Paléolithique supérieur au nord de la Loire. Ce nouveau témoignage, certes plus discret, vient enrichir un corpus en effet peu abondant et l'un de ses intérêts va être de permettre des rapprochements stylistiques avec des objets plus méridionaux de la Charente, de l'Aquitaine ou encore des Pyrénées. En l'état, il est vain de tenter d'aller plus loin dans la description des figures et nous envisageons de confier rapidement ce bâton percé décoré à un restaurateur qui mettra au jour l'ensemble du décor avant sa publication définitive.

\section{RÉFÉRENCES BIBLIOGRAPHIQUES}

SCHMIDER B., VALENTIN B., BAFFIER D., DAVID F., JULIEN M. LEROI-GOURHAN Arl., MOURER-CHAUVIRÉ C., POULAIN T. ROBLIN-JOUVE A., TABORIN Y. (1995) - L'abri du Lagopède (fouilles Leroi-Gourhan) et le Magdalénien des grottes de la Cure (Yonne), Gallia Préhistoire, 37, p. 55-114.

DACHARY M., CHAUVIÈRE F.-X. (2006) - Catalogue des pièces exceptionnelles mises au jour au Grand Pastou, à Dufaure et Duruthy, in M. Dachary dir., Les Magdaléniens à Duruthy. Qui étaient-ils? Comment vivaient-ils? Hastingues, Conseil général des Landes centre départemental du patrimoine, p. 96-186.

FICATIER A. (1886) - Étude paléoethnologique sur la grotte magdalénienne du Trilobite à Arcy-sur-Cure (Yonne), extrait de l'almanach historique de l'Yonne de 1887, Auxerre, Albert Gallot, 25 p.

LEROI-GOURHAN Arl., LEROI-GOURHAN A. (1964) - Chronologie des grottes d'Arcy-sur-Cure (Yonne), Gallia Préhistoire, 7, p. 1-64.

PARAT A. (1902) - Les grottes de la Cure, XXI à XXIV, Bulletin de la Société des sciences historiques et naturelles de l'Yonne, 56, 2, p. 4990.

PARAT A. (1903) - Catalogue du matériel déposé au musée de Joigny, manuscrit conservé au musée de l'Avallonnais.

PELTIER, A. (1992- - Fiche générale bâtons percés, in H. Barge-Mahieu, H. Camps-Fabrer, V. Feruglio, A. Peltier, D. Ramseyer dir., Fiches typologiques de l'industrie osseuse préhistorique, cahier $V$ : bâtons percés, baguettes, Treignes, CEDARC, p. 7-34.

PÉTILLON J.-M. (2006) - Des Magdaléniens en armes. Technologie des armatures de projectile en bois de cervidé du Magdalénien supérieur de la grotte d'Isturitz. (Pyrénées-Atlantiques), Treignes, CEDARC (Artefacts 10), $302 \mathrm{p}$

Danièle MOLEZ

CNRS, Laboratoire ARSCAN (UMR 7041) Maison René Ginouvès

21, allée de l'Université, 92023 Nanterre cedex

Jean-Marc PÉTILLON

CNRS, Laboratoire TRACES (UMR 5608)

Maison de la recherche, Université Toulouse 2 5, allées Antonio-Machado, 31058 Toulouse cedex 9 